\title{
Dielectric and Mechanical Nonlinear Behavior of Mn Doped PMN-35PT Ceramics
}

\author{
Diouma Kobor ${ }^{1}$, Abdelowahed Hajjaji ${ }^{2}$, Jose E. Garcia ${ }^{3}$, Rafel Perez ${ }^{3}$, Alfons Albareda ${ }^{3}$, \\ Laurent Lebrun ${ }^{4}$, Daniel Guyomar ${ }^{4}$ \\ ${ }^{1}$ Laboratoire de Chimie et Physique des Matériaux (LCPM), Université de Ziguinchor, Quartier Diabir, \\ Ziguinchor, Sénégal \\ ${ }^{2}$ Ecole Nationale des Sciences Appliquées d'El Jadida, EL Jadida, Morocco \\ ${ }^{3}$ Departament de Fisica Aplicada, Universitat Politècnica de Catalunya, Jordi Girona 1-3, Campus Nord, \\ Barcelona, Spain \\ ${ }^{4}$ INSA, LGEF, Bat Gustave FERRIE, 8 Rue de la Physique, Villeurbanne Cedex, France \\ E-mail:dkobor@univ-zig.sn
}

Received August 14, 2010; revised September 26, 2010; accepted August 30, 2010

\begin{abstract}
This paper presents an investigation on dielectric and mechanical nonlinear properties in Mn-doped PMN-35PT ceramics. The structural study of the ceramics verifies that the $1 \%$ mol Mn doped PMN-35PT is a pure perovskite phase with a tetragonal symmetry. SEM micrograph shows the same microstructural morphology of an undoped ceramic. From the EPR spectra, it has been concluded that the major part of Mn is present in $\mathrm{Mn}^{2+}$ rather than in $\mathrm{Mn}^{4+}$ form. The addition of $\mathrm{Mn}^{2+}$ ions acts on the dielectric, piezoelectric and mechanical properties by decreasing the relative dielectric permittivity (3800 to 2074), the dielectric losses ( 0.60 to 0.53$)$, the piezoelectric coefficient $\mathrm{d}_{33}(650$ to $403 \mathrm{pC} / \mathrm{N})$, and increasing the mechanical quality factor $\mathrm{Q}_{\mathrm{m}}(78$ to 317$)$. It was found that in $\mathrm{Mn}^{2+}$ doped ceramics the dielectric response can not be described by Rayleigh law. This result can be understood taking into account that reversible motion of the domain wall is a relevant contribution to response of this material.
\end{abstract}

Keywords: Piezoelectrics, Ceramics, Nonlinearity, Dielectric properties, Mechanical Properties, PMN-PT

\section{Introduction}

Since the discovery of relaxor behavior in $\mathrm{Pb}\left(\mathrm{Mg}_{1 / 3} \mathrm{Nb}_{2 / 3}\right) \mathrm{O}_{3}$ (PMN) system by Smolenskii and Agranovskaya [1], many investigations on mixed $\mathrm{B}$-site cation relaxor ferroelectrics, i.e. $\mathrm{Pb}\left(\mathrm{BI}_{1 / 3} \mathrm{BII}_{2 / 3}\right) \mathrm{O}_{3}$-type perovskites, have been performed due to their excellent dielectric and electromechanical properties [2]. Lead magnesium niobate-lead titanate $(1-x)\left[\mathrm{Pb}\left(\mathrm{Mg}_{1 / 3} \mathrm{Nb}_{2 / 3}\right) \mathrm{O}_{3}\right]-x \mathrm{PbTiO}_{3}$ (PMN-PT) is a solid solution of relaxor PMN and normal $\mathrm{PbTiO}_{3}$ (PT) ferroelectrics which represent a typical example of such perovskites. These materials with composition close to the morphotropic phase boundary (MPB) exhibit unusually high electromechanical coupling coefficients and electrically induced strain [3]. The investigation of compositions near the MPB region has attracted particular attention owing to their excellent piezoelectric and dielectric properties, which make them promising candidate materials for applications in multilayer ce- ramic capacitors, as well as ceramic electrostrictive actuators and field-induced electromechanical transducers [3-5], which are often operated at the high field regime [6]. They are also very attractive to improve high performance actuators and sensors design in advanced applications such as image ultrasonic system and sonar. Unfortunately piezoceramics show a pronounced nonlinearity [7]. This phenomenon has a rather complex physical origin. Nonlinearities appear as alterations of elastic, electric and piezoelectric behavior [8]. Therefore, many applications can benefit from a better understanding of the ceramics performance under various electrical, mechanical and temperature conditions. In this context, several phenomenological models have been proposed to explain the physical origin of the observed dielectric, mechanical and piezoelectric anomalies [9]. Nevertheless, none of the models has gained universal acceptance yet, due to some contradictory experimental results, as for example the different behavior observed in soft and hard 
lead zirconate titanate ceramics (PZT).

PMN-PT system shows, from the fundamental point of view, intriguing physical properties, which are reflected in the dielectric response. However, in this system, there are ambiguous reports concerning the existence and origin of the observed dielectric behavior under external applied electric fields [10], although it is widely accepted that one of their origin is the movement of the domain walls [9]. In a recent work, dielectric and piezoelectric nonlinear responses of PMN-35PT ceramics were investigated [11]. Results showed typical soft-type behaviour, with high linear properties (dielectric and piezoelectric coefficients). However, it is necessary to improve the performance of this system for typical hard-type piezoceramics applications. In this sense, the objective of this work is to study and to understand the effect of Mn doping on PMN-35PT in order to improve the dielectric and mechanical responses of this system for high power application.

\section{Experimental Procedure}

$0.65\left[\mathrm{~Pb}\left(\mathrm{Mg}_{1 / 2} \mathrm{Nb}_{2 / 3}\right)\right] \mathrm{O}_{3}-0.35 \mathrm{PbTiO}_{3}$ ceramics doped with $1 \% \mathrm{Mn}$, hereafter labeled as PMN-35PT $+1 \% \mathrm{Mn}$, were prepared by using fine powders, as previously reported [12]. High purity chemical powders $\mathrm{PbO}(99 \%$ QS), $\mathrm{TiO}_{2}$ (99\% Merck), $\mathrm{MgO}$ (99\% Merck), $\mathrm{Nb}_{2} \mathrm{O}_{5}$ (99.9\% Aldrich) were used as starting materials. The PMN-PT ceramics were synthesized using the two-step columbite precursor technique. Powders of Magnesium oxide $(\mathrm{MgO})$ and Niobium oxide $\left(\mathrm{Nb}_{2} \mathrm{O}_{5}\right)$ were mixed in alcohol, using $\mathrm{MgO}$ excess, ground and calcined at $1100^{\circ} \mathrm{C}$ for 8 hours in order to synthesize $\mathrm{MgNb}_{2} \mathrm{O}_{6}$ phase. Magnesium niobate was mixed with the $\mathrm{MnO}_{2}$, $\mathrm{PbO}$ and $\mathrm{TiO}_{2}$ in the ratio of PMN-PT 65-35 in molar percentage. Small excess of $\mathrm{PbO}$ was added to compensate its loss during heat treatments. This mixture was calcined at $825^{\circ} \mathrm{C}$ for 2 hours. The resulting mixture of $1 \% \mathrm{Mn}+\mathrm{PMN}-\mathrm{PT}$ powder was mixed with $10 \mathrm{wt} \%$ polyvinyl alcohol (PVA) solution, and was pressed to prepare pellets with $13 \mathrm{~mm}$ of diameter and 1,2 $\mathrm{mm}$ of thickness with a $80 \mathrm{MPa}$ uniaxial stress. The pellets were first heated at $600^{\circ} \mathrm{C}$ to burn out the PVA, and then sin- tered at $1250^{\circ} \mathrm{C}$ for 10 hours in a sealed alumina crucible with $5 \mathrm{~g}$ of $\mathrm{PbZrO}_{3}$ to prevent lead losses during sintering. A pure perovskite phase of $1 \% \mathrm{Mn}$ doped PMN-PT was obtained.

With this amount of dopant, polycrystalline PZT ceramics show a high increase of quality factor $\left(Q_{m}\right)$ without significantly decreasing the piezoelectric coefficients [13]. X-ray powder diffraction was performed on sintered ceramics with the help of a Philips X'pert Pro diffractometer using a monochromatic $\mathrm{CuK}_{\alpha}$ radiation $(\lambda=$ $1.5418 \AA$ A). Scanning electronic microscopy techniques was carried out by using a JMS 840 (JEOL). An EPR VARIAN E19 spectrometer was used to determine the Manganese valences.

The samples were obtained in the form of discs with $13 \mathrm{~mm}$ of diameter and $1 \mathrm{~mm}$ of thickness. Parallel faces were sputtered with silver for functional properties measurements. Poling was performed with an electric field of $3 \mathrm{kV} / \mathrm{cm}$ during 30 minutes at room temperature. Dielectric properties were determined as a function of temperature by using a multi-frequency LCR meter (HP4284A) and a computer-controlled temperature chamber. From these plots (not published), the phase transition temperature $\left(\mathrm{T}_{\mathrm{c}}\right)$ and the room temperature dielectric properties were identified. The longitudinal mode piezoelectric coefficient $d_{33}$ was measured with the help of a Berlincourt $d_{33}$ meter. Electromechanical properties were calculated from the resonance peak measurement.

Table 1 summarizes the dielectric, piezoelectric and electromechanical coefficients of undoped and $1 \% \mathrm{Mn}$ doped PMN-35PT ceramics. The dielectric and piezoelectric properties decrease with the doping while the mechanical quality factor $\mathrm{Q}_{\mathrm{m}}$ increases largely (78 to 317 ). Such behavior is found in literature for hard ceramics [3]. This fact suggests that $\mathrm{Mn}$ addition induces some mechanisms that turn the PMN-PT properties from soft- to hard-type.

\subsection{Dielectric and Mechanical Nonlinearities}

The non-linear dielectric measurements are made at 1 $\mathrm{kHz}$, by a modified comparison capacitance bridge that

Table 1. Dielectric, piezoelectric and electromechanical properties of undoped and 1\% Mn doped PMN-35PT ceramics at room temperature. Curie temperatures are also reported. Dielectric measurements have been carried out at $1 \mathrm{kHz}$.

\begin{tabular}{|c|c|c|c|c|c|c|c|c|}
\hline & $\begin{array}{c}d_{33} \\
(\mathrm{pC} / \mathrm{N})\end{array}$ & $\mathbf{K}_{\mathbf{P}}$ & $\mathbf{K}_{\mathbf{t}}$ & $\begin{array}{c}S^{E_{11}} \\
\left(10^{-12} \mathbf{m}^{2} / \mathbf{N}\right)\end{array}$ & $\varepsilon_{\mathrm{L}}$ & $\tan \delta_{\mathrm{e}}(\%)$ & $\mathbf{Q}_{\mathbf{m}}$ & $\mathrm{T}_{\mathrm{c}}\left({ }^{\circ} \mathrm{C}\right)$ \\
\hline \multirow{2}{*}{ PMN-35PT } & $650[5]$ & $0,50[3]$ & $0,57[5]$ & $13,30[\mathbf{1}]$ & 3800 [1] & $0,60[1]$ & $78[4]$ & $165[1]$ \\
\hline & $595[3]$ & & $0,60[2]$ & $19,00[4]$ & 4220 [5] & $1,96[3]$ & 78 [5] & $170[6]$ \\
\hline \multirow{2}{*}{ PMN-35PT+1\%Mn } & 405 & 0,49 & 0,51 & 10,30 & 2074 & 0,53 & 317 & 175 \\
\hline & $455[3]$ & $0,48[3]$ & - & $11,00[4]$ & $2100[4]$ & $0,78[3]$ & $300[4]$ & $175[4]$ \\
\hline
\end{tabular}


is compensated at low signal in order to obtain the nonlinear contribution to the electric displacement $D_{\mathrm{NL}}(E)$, and consequently the nonlinear dielectric constant increment, $\Delta \varepsilon_{\mathrm{NL}}\left(E, E_{0}\right)$, as a function of instantaneous electric field, $E$. To prevent overheating of the ceramics due to the high signal excitation, burst signal excitations with only 5 cycles are used. After subtraction of the antisymmetric contribution $\varepsilon_{\mathrm{A}}$ (a low contribution that depends on the polarization), the symmetric contribution $\varepsilon_{S}$ is analyzed. This term is divided again into two contributions: the first, $\varepsilon_{\alpha}$, depends only to the electric field amplitude, and the second, $\varepsilon_{\beta}$, is the remaining contribution, which depends on the instantaneous electric field, as is detailed in Ref. [14]. The increment of dielectric constant has been written as follow:

$$
\Delta \varepsilon_{\mathrm{NL}}\left(\mathrm{E}, \mathrm{E}_{0}\right)=\varepsilon_{\mathrm{A}}\left(\mathrm{E}, \mathrm{E}_{0}\right)+\varepsilon_{\mathrm{S}}\left(\mathrm{E}, \mathrm{E}_{0}\right)=\varepsilon_{\mathrm{A}}\left(\mathrm{E}, \mathrm{E}_{0}\right)+\varepsilon_{\alpha}\left(\mathrm{E}_{0}\right)
$$$$
+\varepsilon_{\beta}\left(\mathrm{E}, \mathrm{E}_{0}\right) \text {. }
$$

For PZT based piezoceramics, the contribution $\varepsilon_{\alpha}$ has been satisfactorily associated to irreversible contribution of domain wall motion, while $\varepsilon_{\beta}$ represents a reversible contribution which has been related to the bending of domain walls [15].

Measurements of mechanical nonlinearities have been obtained by exciting the 1st radial mode of resonance. Bursts of different amplitudes and equal frequency, close to the resonant frequency, were applied, by measuring the electrical impedance [16]. The increase of the real part of impedance are related to the increase of mechanical losses $\Delta \operatorname{tg}(\delta \mathrm{m})$, while the increase of the imaginary part is related to the shift of the resonant frequency, and thus it is related to the increase of the compliance $s_{11}^{E}$. The vibration velocity of the border of the disc $v(R)$ has also been measured by using a laser vibrometer, in order to obtain the mean strain $\langle S\rangle$.

From these measurements it is possible to obtain the values of the motional electrical displacement $D_{\mathrm{m}}=$ $I_{\mathrm{m}} / A \omega$, and the main stress $T$, defined as follows:

$$
\begin{aligned}
& \langle S\rangle=\left\langle S_{r}+S_{\theta}\right\rangle=\frac{2 v(R)}{R \omega}, \\
& T=\left\langle T_{r}+T_{\theta}\right\rangle=\frac{\langle S\rangle}{S_{11}^{E}(1-\sigma)} .
\end{aligned}
$$

Finally, the piezoelectric coefficient $d_{31}$ is obtained as a ratio between dielectric displacement and the main stress, $d_{31}=D_{m} / T$, assuming that in the resonance the electric field is null.

\section{Results and Discussions}

\subsection{Structural Characterization}

Figure 1 shows the XRD diffraction patterns, which con- firm a pure perovskite phase with a majority tetragonal symmetry for PMN-PT $+1 \% \mathrm{Mn}$. No residual phases are detected. Generally, XRD diagrams are carried out over narrow angular regions centered about the six pseudo-cubic reflections (100), (110), (111), (200), (220) and (222), from which it is possible to determine unambiguously the crystal's symmetry [17]. It is clear that the tetragonal symmetry is characterized by the presence of the (h00) doubled peaks. In our case, from the peaks profiles of the (100) and (200) reflections, the symmetry is clearly seen to be tetragonal with a possible presence of a rhombohedral symmetry. This structure is the same that recently has been published by Guerra et al. for PMN-35PT ceramics [10].

These results show that the $1 \%$ mol Mn doping didn't change the PMN-35PT ceramics structure at room temperature.

SEM images revealed a homogeneous grain size distribution and morphology for PMN-35PT $+1 \% \mathrm{Mn}$ as can be shown in Figure 2. This image confirms that there exists no pyrochlore phase grain, which is characterized by its pyramidal form. The micro-structural morphology presents an average grain size between 3 to 8 $\mu \mathrm{m}$ for the undoped PMN-35PT ceramics [12] and 6 to 8 $\mu \mathrm{m}$ for the Mn doped ones.

Figure 3 shows the EPR spectrum for PMN-35PT + $1 \% \mathrm{Mn}$. Two peaks are detected at about 1800 and 3200 $\mathrm{G}$ which are attributed, respectively, to the $\mathrm{Mn}^{4+}$ and $\mathrm{Mn}^{2+}$ ions, confirming that the major part of $\mathrm{Mn}$ is at its lower valence $2+$. The presence of $\mathrm{Mn}^{4+}$ can be seen, while it is impossible to prove with this technique the presence of $\mathrm{Mn}^{3+}$ which has an anti-parallel spin. The $\mathrm{Mn}^{2+}$ peak forms a sextet. This is due to the hyperfine interaction in the structure for which the most important contributor is the spin-orbit coupling. These 6 hyperfine lines are due to the degenerated energetic states $\left(\mathrm{m}_{\mathrm{I}}=5 / 2\right.$, $3 / 2,1 / 2,-1 / 2,-3 / 2$ and $-5 / 2$ )

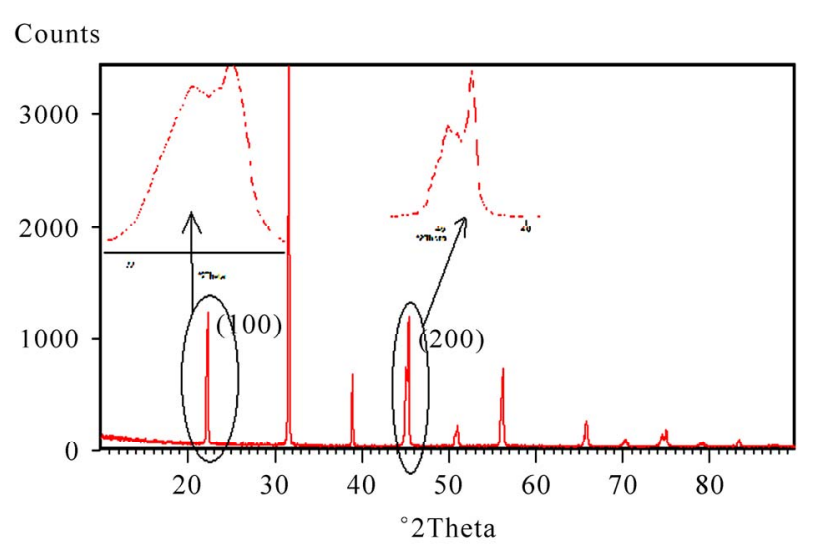

Figure 1. X-Ray Diffraction patterns of $1 \%$ mol Mn doped PMN-35PT ceramics. 


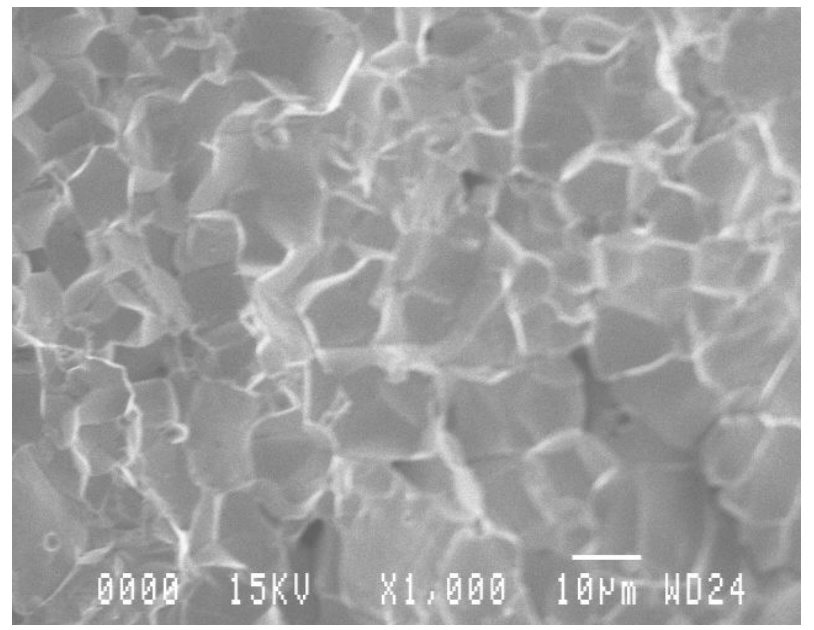

Figure 2. SEM images of $1 \%$ mol Mn doped PMN-35PT ceramics.

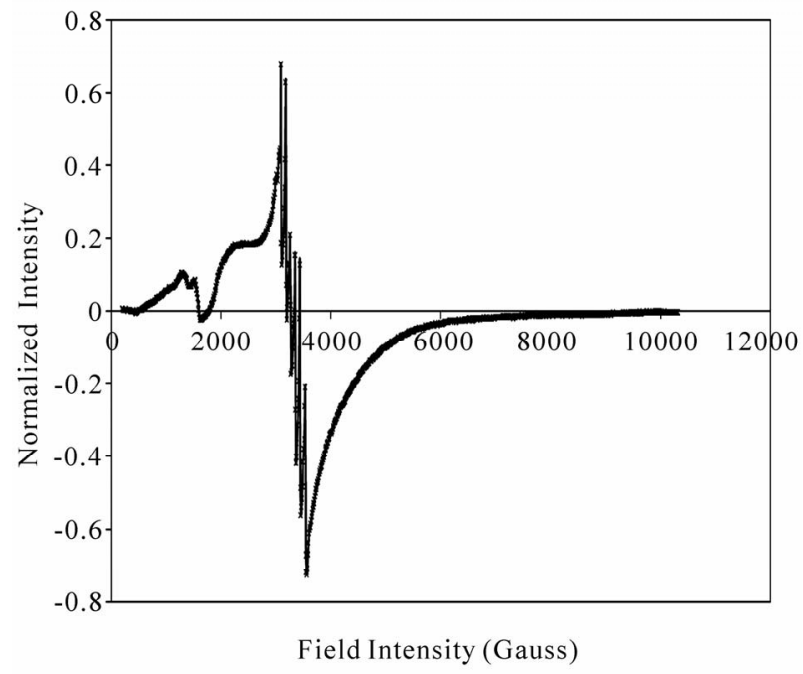

Figure 3. EPR spectrum of $1 \%$ mol Mn doped PMN-35PT ceramics.

\subsection{Dielectric and Mechanical Nonlinearities}

Dielectric and piezoelectric responses of ferroelectric materials are due to the addition of two contributions, denoted in the literature as intrinsic and extrinsic. Intrinsic contribution is related to the change of the unit cell polarization and it is the only effect that can be observed in a monodomain-like structure system. The extrinsic contribution is considered as the response of different phenomena, as the motion of the domain walls and their interaction with the lattice defects [15]. The dielectric nonlinearities of ferroelectric materials are intimately related to extrinsic effects [18]. The dielectric non-linearity in the undoped PMN-35PT is adequately described by the Rayleigh model [11]. According to the model, the response of the material is produced by the irreversible motion of the domain walls. As a consequence, the dielectric constant $\varepsilon$ ' linearly depends on the electric field amplitude, and the ratio between the losses and the dielectric constant has a constant value equal to $\varepsilon^{\prime \prime} / \varepsilon^{\prime}=0.42$. $[15,18]$. In terms of the equation 1 , this model implies that $\varepsilon_{\beta}=0$, as it is verified in the undoped PMN-35PT.

Figure 4 shows the dependence of the dielectric constant with applied electric field amplitude, $\mathrm{E}_{0}$, for PMN-35PT + 1\%Mn.

The observed behavior for this material is completely different from the behavior of the undoped PMN-35PT. In this case, dielectric constant does not follow a linear relation with $\mathrm{E}_{0}$ as is expected by the Rayleigh law.

A polynomial function can be used for modeling the relationship:

$$
\varepsilon_{\mathrm{r}}\left(\mathrm{E}_{0}\right)=\varepsilon_{\mathrm{L}}+\alpha \mathrm{E}_{0}+\beta \mathrm{E}_{0}^{2}
$$

where $\varepsilon_{\mathrm{L}}$ is the dielectric constant at zero field (linear dielectric constant), and $\alpha$ and $\beta$ are constants depending on the material type.

On Rayleigh model, the value of $\beta$ must be null, and $\alpha$ is a positive value that measures the strength of the non linear effect. For PMN-35PT $+1 \% \mathrm{Mn}$, the calculated values are: $\alpha=-1170 \mathrm{~m} / \mathrm{MV}$ and $\beta=28734 \mathrm{~m}^{2} /(\mathrm{MV})^{2}$, indicating that Rayleigh law is not applicable to PMN-35PT $+1 \% \mathrm{Mn}$. The value of $\varepsilon_{\mathrm{L}}$ found applying the Equation (1)'s fitting is 2097 which is quite equal to the value of the relative dielectric permittivity constant measured at $1 \mathrm{kHz}$ (2074).

Domain wall contributions to dielectric response can be investigated by plotting $\varepsilon$ "vs. $\varepsilon$ ' and $\varepsilon$ " vs. $\varepsilon_{\alpha}$ As can be shown in Figure 5, a linear behavior was obtained for the second relation. For undoped PMN-35PT ceramics, the ratio between the increment of $\varepsilon$ "and $\varepsilon_{\alpha}\left(m_{\alpha}\right)$ and the ratio between the increment of $\varepsilon^{\prime \prime}$ and $\varepsilon^{\prime}\left(m_{\varepsilon}\right)$ are the

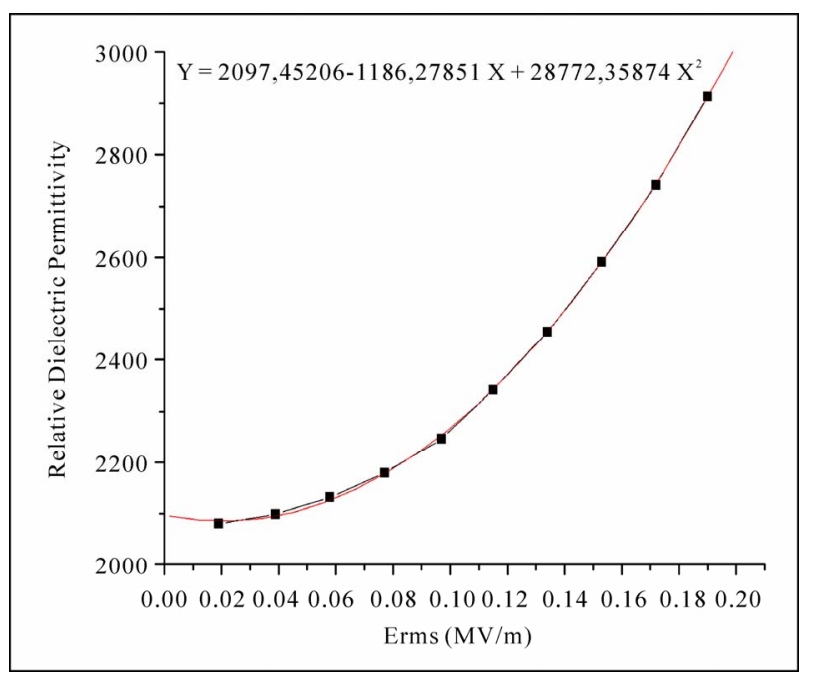

Figure 4. Relative Dielectric Permittivity versus $E_{r m s}$ of $1 \%$ mol Mn doped PMN-35PT ceramics. 


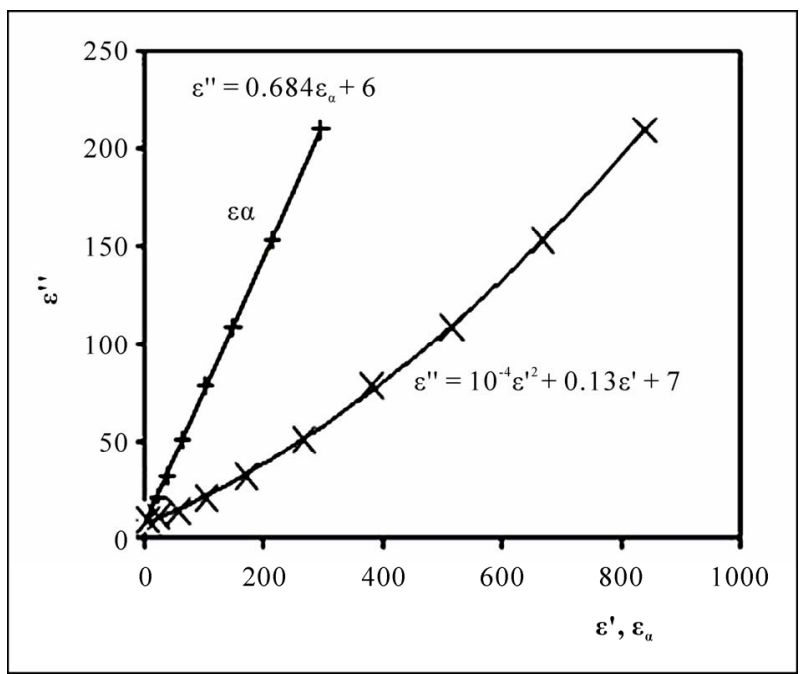

Figure 5. The permittivity imaginary part as a fonction of the real part $\Delta \varepsilon$ ' and the $\varepsilon_{\alpha}$ of $1 \%$ mol Mn doped PMN-35PT ceramics.

same $m_{\alpha} \approx m_{\varepsilon}=0.70$ [17]. In our case $\varepsilon$ " versus $\varepsilon$ ' follows a quadratic relation. Such behavior is completely different to that obtained for undoped PMN-35PT ceramics, in which both the two relations are linear. It is interesting to note that for PMN-35PT $+1 \% \mathrm{Mn}$, the dielectric losses doesn't vary linearly with the dielectric permittivity. Nevertheless, the value of $m_{\alpha}(0,68)$ is quite the same for the two materials.

When these values are not equal $m_{\alpha} \neq m_{\varepsilon}$, we expect that the mechanism that produces the dielectric permittivity variation depends on both reversible and irreversible contributions [15].

Furthermore, the decrease of $m_{\varepsilon}$ from 0.70 to 0.24 (value measured by extrapolating the curve as a linear one) strongly suggests that the reversible contribution $\left(\varepsilon_{\beta}\right)$ of the dielectric permittivity becomes relevant and can not be neglected for Mn doped PMN-35PT ceramics. This behavior is similar to that observed in hard-type PZT, indicating that $\mathrm{Mn}^{2+}$ substitution is a good way to improve PMN-PT properties for transducer applications.

These results can be understood as a consequence of the substitution of high valence ions by low valence ions in the PMN-PT structure. The addition of $\mathrm{Mn}^{2+}$ ions in the perovskite $\mathrm{B}$ sites produce oxygen vacancies, giving rise to the creation of complex defects, which acts as pinning centers that reduces the mobility of the domain wall. As a consequence, in the PMN-PT $+1 \% \mathrm{Mn}$ the non linearity has less significance than in the undoped PMN-PT. Moreover, the reversible contribution to the domain wall motion is an important mechanism in the first case but it is negligible in the second. In conclusion, the two rela- tions can be written as following:

$$
\varepsilon^{\prime \prime}=0.68 \varepsilon_{\alpha}+6
$$

$$
\varepsilon^{\prime \prime}=10^{-4} \varepsilon^{2}+0.13 \varepsilon^{\prime}+7
$$

Figure 6 shows the dependence of the piezoelectric coefficient $d_{31}$ and the compliance $s^{\mathrm{E}}{ }_{11}$ versus the stress $T$. These plots show that $d_{31}$ and $s^{\mathrm{E}}{ }_{11}$ does not increase linearly with $T$. It is important to note that these relations are nearly the same that those found in the dielectric response. Mechanical and dielectric properties are in the similar polynomial dependence with the stress $T$ or the field amplitude $E_{0}$. These results permit to conclude the existence of interdependence between the origins of the phenomena related to these two responses.

Figure 7 shows the imaginary part $X$ versus the real part $R$ of the impedance $Z$ when the current rises. It has a

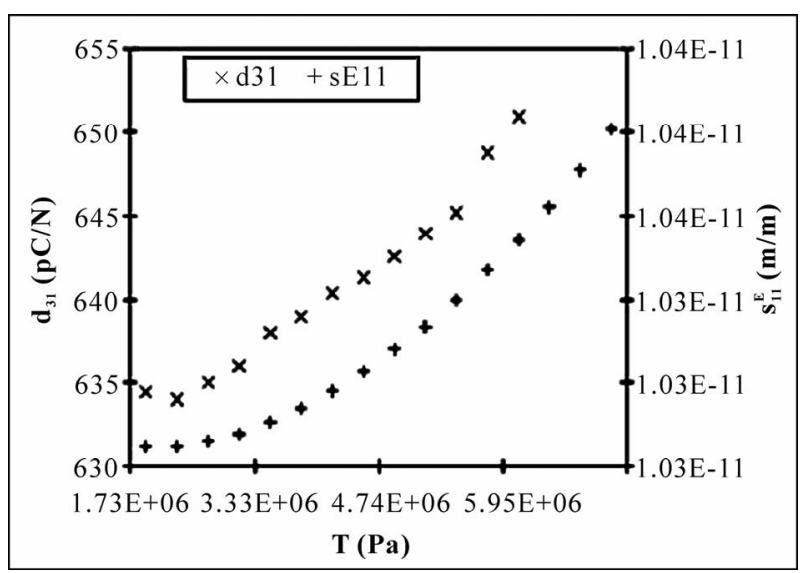

Figure 6. d31 and SE11 dependance of the stress T of $1 \%$ mol Mn doped PMN-35PT ceramics.

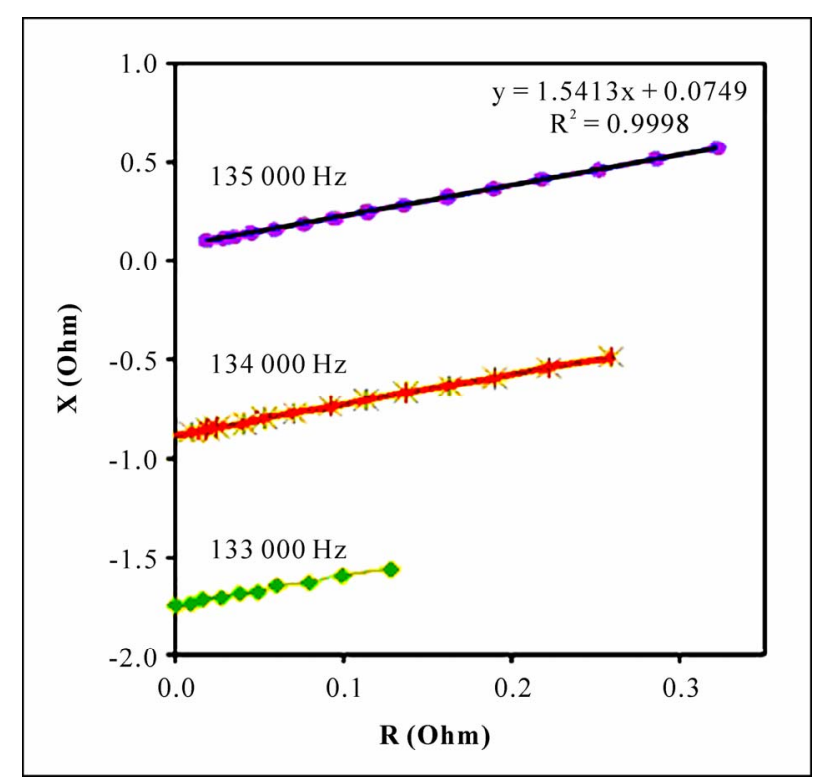

Figure 7. The imaginary part $X$ versus the real $R$ of the impedance $Z$ at different frequencies for $1 \% \mathrm{~mol} M n$ doped PMN-35PT ceramics. 
linear dependence with a slope $m$ around $3 / 2$. The inverse of this slope, $1 / m$, is the same as the value of $m_{\alpha}$ found in Figure $5(2 / 3=0.67)$. This relationship between these two properties shows that there is an interdependence of the phenomena that lie behind them.

\section{Conclusion}

In this study, the effect of Mn doping on the structural and non-linear dielectric and mechanical properties for PMN-35PT ceramics is shown. It can be concluded that $1 \%$ mol Mn doping does not change the phase structure and grain morphology of PMN-35PT ceramics. EPR studies show that Manganese was present in majority in its lower valence $2+$, achieving a large increase of electromechanical quality factor while keeping good values of the dielectric and piezoelectric properties. The Mn doped ceramics do not follow the Rayleigh law, indicating that in this case the reversible movement of domain wall is a relevant contribution to material response. Results of this work demonstrate that Mn doping is a good way to obtain new compounds of PMN-PT systems with better properties for high power applications.

\section{References}

[1] G. A. Smolenskii and A. I. Agranovskaya, "Dielectric Polarization and Losses of Some Complex Compounds," Soviet Physics Technical Physics, Vol. 3, No. 3, 1958, pp. 1380-1389.

[2] Y. H. Chen, K. Uchino and D. Viehland, "Substituent Effects in $0.65 \mathrm{~Pb}(\mathrm{Mg} 1 / 3 \mathrm{Nb} 2 / 3) \mathrm{O} 3-0.35 \mathrm{PbTiO} 3 \mathrm{Ceram}-$ ics," Journal of Electroceramics, Vol. 6, No. 1, 2001, pp. 13-17.

[3] M. E. Lines and G. M. Glass, "Principles and Applications of Ferroelectrics and Related Materials," Oxford University Press, Oxford, United Kingdom, 2001, p. 77.

[4] S. E. Park and T. R. Shrout, "Ultrahigh Strain and Piezoelectric Behavior in Relaxor Based Ferroelectric Single Crystals,' Journal of Applied Physics, Vol. 82, No. 4, 1997, pp. 1804-1811.

[5] S. W. Choi, T. R. Shrout, S. J. Jang and A. S. Bhalla, "Morphotropic Phase Boundary in $\mathrm{Pb}(\mathrm{Mgl} / 3 \mathrm{Nb} 2 / 3-$ PbTiO3 System," Materials Letters, Vol. 8, No. 6-7, 1989, pp. 253-255.

[6] H. Takagi, K. Sakata and T. Takenaka, "Electrostrictive Properties of $\mathrm{Pb}\left(\mathrm{Mg}_{1 / 3} \mathrm{Nb}_{2 / 3}\right) \mathrm{O}_{3}$-Based Relaxor Ferroelectric Ceramics," Japanese Journal of Applied Physics, Vol. 32, No. 9S, 1993, pp. 4280-4283.

[7] D. A. Hall, "Nonlinearity in Piezoelectric Ceramics," Journal of Material Sciences, Vol. 36, No. 19, 2001, pp.
4575-4601.

[8] D. Kobor, A. Albareda, R. Perez, J. Garcia, L. Lebrun and D. Guyomar, "Dielectric and Mechanical Nonlinearities of $<001>$ Oriented Pure and Doped Single Crystals of PZN-4.5PT," Journal of Physics D: Applied Physics, Vol. 38, No. 13, 2005, pp. 2258-2264.

[9] A. K. Tangsev and A. E. Glazounov, "Mechanism of Polarization Response in the Ergodic Phase of a Relaxor Ferroelectric,'" Physical Review B, Vol. 57, No. 1, 1998, pp. 18-21.

[10] J. D. S. Guerra, E. B. Araújo, C. A. Guarany, R. N. Reis and E. C. Lima, "Features of Dielectric Response in PMN-PT Ferroelectric Ceramics," Journal of Physics D: Applied Physics, Vol. 41, No. 22, 2008, pp. 225504225508.

[11] J. E. Garcia, J. D. S. Guerra, E. B. Araújo and R. Pérez, "Domain Wall Contribution to Dielectric and Piezoelectric Responses in $0.65 \mathrm{~Pb}\left(\mathrm{Mg}_{1 / 3} \mathrm{Nb}_{2 / 3}\right)-0.35 \mathrm{PbTiO}_{3}$ FerroEectric Ceramics,' Journal of Physics D: Applied Physics, Vol. 42, No. 11, 2009, pp. 115421-115425.

[12] A. Benayad, "Matériaux Monocristallins à Forte Activité Piézoélectrique: élaboration, Caractérisation et Application," Thesis, INSA, December 2005, p. 155.

[13] L. Lebrun, B. Guiffard, E. Boucher, D. Audigier, D. Guyomar, L. Eyraud and E. Pleska, "Dielectric and Piezoelectric Properties of ( $\mathrm{La}, \mathrm{Mg}, \mathrm{F})$ and $(\mathrm{Mg}, \mathrm{Mn}, \mathrm{F})$ Doped PZT Ceramics under Low and High Solicitations," Journal of the European Ceramic Society, Vol. 21, No. 10-11, 2001, pp. 1357-1360.

[14] J. E. Garcia, R. Pérez and A. Albareda, "High Electric Field Measurement of Dielectric Constant and Losses of Ferroelectric Ceramics,' Journal of Physics D: Applied Physics, Vol. 34, No. 22, 2001, pp. 3279-3284.

[15] J. E. Garcia, R. Pérez and A. Albareda, "Contribution of Reversible Processes to the Non-Linear Dielectric Response in Hard Lead Zirconate Titanate Ceramics," Journal of Physics: Condensed Matter, Vol. 17, No. 44, 2005, pp. 7143-7150.

[16] J. A. Casals, A. Albareda, R. Pérez, J. E. García, E. Minguella and F. M. de Espinosa, "Nonlinear Characterization with Burst Excitation of 1-3 Piezocomposites Transducers," Ultrasonics, Vol. 41, No. 4, 2003, pp. $307-$ 311.

[17] A. Benayad, D. Kobor, L. Lebrun, B. Guiffard and D. Guyomar, "Characteristics of $\mathrm{Pb}\left[\left(\mathrm{Zn}_{1 / 3} \mathrm{Nb}_{2 / 3}\right)_{0.955} \mathrm{Ti}_{0.045}\right]$ $\mathrm{O}_{3}$ Single Crystals Versus Growth Method," Journal of Crystal Growth, Vol. 270, No. 1-2, 2004, pp. 137-144.

[18] D. Damjanovic and M. Demartin, "Contribution of the Irreversible Displacement of Domain Walls to the Piezoelectric Effect in Barium Titanate and Lead Zirconate Titanate Ceramics," Journal of Physics: Condensed Matter, Vol. 9, No. 23, 1997, pp. 4943-4953. 